\title{
Breaking biological borders
}

\section{Opportunities in nanotechnology are opening up in Japan - especially for young researchers willing to cooperate across disciplines, says Robert Triendl.}

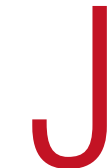

apan's love affair with nanotechnology has, until recently, tended to centre on physics. Most of Japan's nanotech funding, which has been supported by a number of agencies since the 1980s, has remained oriented towards studying nanoscale phenomena in semiconductor materials or developing new materials.

But that is changing. In recent months, policymakers have been strengthening research capacity at the interface between life sciences and nanotechnology. Research centres in the public sector are now increasingly offering positions for scientists specializing in nanobiology. And, perhaps most importantly, funding schemes for young scientists in nanobiotechnology are emerging

\section{YOUTH MOVEMENT}

The Japan Science and Technology Corporation funds emerging research areas through schemes such as ERATO, CREST and PRESTO. ERATO (Exploratory Research for Advanced Technology) provides relatively young scientists with a sizeable amount of money typically between US $\$ 15$ million and US $\$ 18$ million over five years - and almost total freedom to spend it.

Toshio Yanagida and his group at Osaka University are one of ERATO's success stories. The experimental techniques they designed during their studies of how 'biomotors' work inside muscle cells have helped to revolutionize the fields of single-molecule imaging and the study of motor proteins. Today, some five years

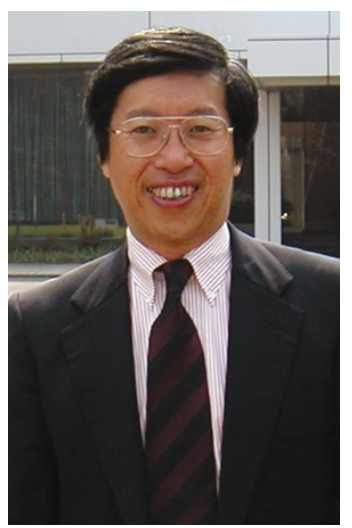
after the project ended, many of the young scientists hired by Yanagida have moved to universities or research institutes across Japan, taking with them knowledge of the techniques developed in Yanagida's lab.

The development of such techniques appears to be one of the brightest areas for employment opportunities in nanobiotechnology. At the Nanotechnology Research Institute (NRI) in Tsukuba, the main research focus is on molecular nanotechnology for applications such as molecular devices or drug-delivery systems. Hiroshi Yokoyama, the NRI's director, says that he plans to hire more biologists. Other institutes, including the new Tissue Engineering Research Center in Osaka, are also strengthening interdisciplinary research in nanobiology.

According to Yokoyama, some 10\% of research funding at his institute is spent on the development of new research tools. "Instrumentation tools provide the basic infrastructure for research in nanotechnology and a platform for cooperation," he says.

Satoshi Kawata, a professor of applied physics at Osaka University, agrees that instrumentation is crucial for nanotechnology. Many tools used by nanotech scientists, such as the atomic force microscope, were originally developed by physicists to study fundamental physical phenomena or the physical properties of new materials. But, says Kawata, it is equally important to develop organizational models that allow cooperation between scientists designing the new techniques and those who will use them, such as cell biologists.

\section{ORGANIZING COOPERATION}

At universities, venture-business laboratories and interdisciplinary research centres are offering space for the development of cooperative endeavours by researchers from various disciplines — although sometimes with mixed results. Osaka University's Frontier Research Center goes a step further.

Set up late last year with government support, the centre aims to provide a better organizational mechanism to fund high-quality research in rapidly emerging areas and to aid cooperation with industry.

But despite such efforts, cooperation between physicists and biologists remains limited. According to Yokoyama, the barriers between disciplines in the nanotechnology field are only slowly breaking down. "Japan's academic circles remain very closed," he says. Robert Triendl is a freelance writer based in Tokyo. ERATO www.jst.go.jp/erato

Japan Science and Technology Corporation www.jst.go.jp/EN Nanotechnology Research Institute unit.aist.go.jp/nanotech Tissue Engineering Research Institute unit.aist.go.jp/terc Frontier Research Center www.frc.handai.com

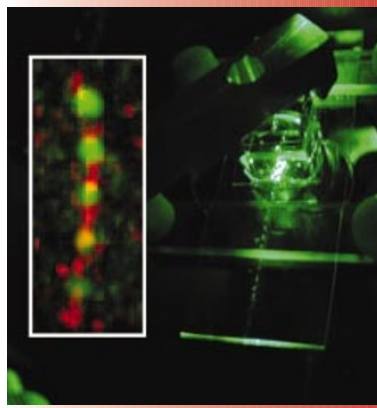

Toshio Yanagida (below) is a pioneer in techniques and instruments (above) for imaging single molecules such as myosin (inset).

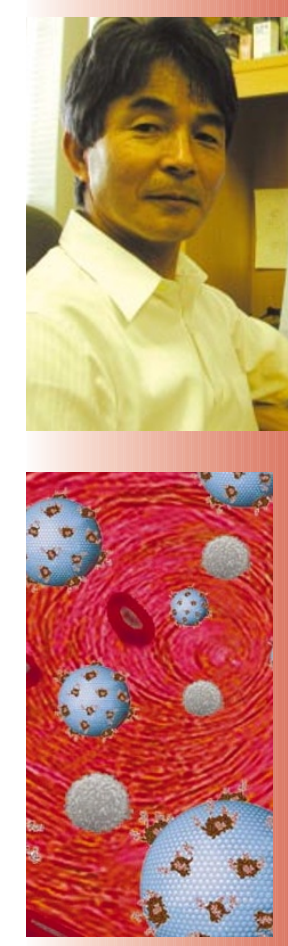

On target: this drugdelivery system, designed in Hiroshi Yokoyama's lab, uses sugar-protein conjugates as receptors. 